\title{
El uso de la tecnología en el tenis moderno: una mirada a la práctica del mejor tenista mundial
}

\section{Dario Novak y Magnus Norman \\ Universidad de Zagreb, Facultad de Kinesiología}

\author{
Palabras clave: tecnología, \\ analisis, GPS \\ Recibido: 15 Marzo 2017 \\ Aceptado: 28 Julio 2017 \\ Autor correspondiente: Dario \\ Novak. Universidad de Zagreb, \\ Facultad de Kinesiología \\ Correo electrónico: \\ dario.novak@kif.hr
}

\section{INTRODUCCIÓN}

El entrenamiento de tenis en cancha y el juego de partidos requieren prolongados y demandantes perfiles de actividad física, que elevan de manera sustancial la tensión fisiológica y perceptual y reducen la función contráctil. Los tenistas deben estar en condición óptima para un gran número de torneos durante el año y no hay tiempo para un "largo" período de preparación (Duffield y cols., 2014). Es por eso que son muy beneficiosas las mediciones que proporcionan una rápida retroalimentación sobre el rendimiento. La identificación temprana de la alta carga de entrenamiento es crítica para evitar el sobre entrenamiento (Hagger y Chatzisarantis, 2005). Hay muchos dispositivos de monitoreo para el deporte elite, y muchos entrenadores los utilizan para mejorar el rendimiento y mitigar el riesgo de lesiones. En general, utilizar la tecnología puede ser muy útil para el análisis del rendimiento deportivo y para que los entrenadores mejoren la calidad de la retroalimentación que entregan a sus jugadores/ atletas, además de incrementar la precisión en las mediciones de tiempo del rendimiento deportivo. Sin embargo, pocos estudios hasta ahora se han focalizado en cuantificar los perfiles durante la práctica de los mejores tenistas. En este estudio los experimentos investigaron (1) los perfiles de actividad de un tenista de alto nivel, en períodos de práctica de aproximadamente 30 minutos (2) la intensidad de la actividad durante períodos pre-definidos de práctica durante el juego de partidos.

\section{MÉTODOS Y PROCEDIMIENTOS}

El objeto de este estudio fue un tenista de elite, masculino, que estaba entre los 10 mejores del mundo, en el momento de llevar a cabo este estudio (Lista de clasificación de profesionales, diciembre de 2016). Prestó su consentimiento, conforme a los requerimientos de la Declaración de Helsinki. El estudio se realizó fuera de temporada, en invierno (noviembre y diciembre de 2016). El período de preparación se caracterizó por una gran cantidad de entrenamiento de acondicionamiento (es decir, correr, entrenamiento de resistencia, tenis, fuerza, y potencia).

El movimiento se grabó utilizando una unidad GPS (OptimEye S5, Catapult Innovations, Melbourne, Australia). La unidad GPS 
incluyó un acelero-medidor tri-axial y un muestreo de giroscopio a $100 \mathrm{~Hz}$, que proporcionaron la información sobre la carga y volumen de los esfuerzos explosivos realizados por el jugador. La unidad se colocó en un pequeño chaleco, en la parte posterior superior del jugador.

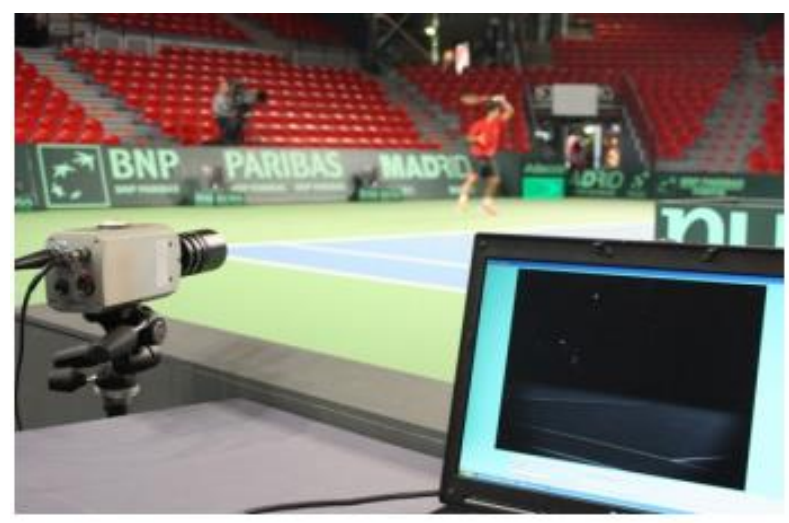

\section{RESULTADOS}

La Tabla 1 muestra el resumen del análisis tiempo-movimiento. En general, las cifras parecen ser muy constantes durante el período observado. Se observa una carga total del jugador de 133 unidades durante el período de 30 minutos, con una carga promedio del jugador por minuto de 4,75 unidades. Es interesante destacar que

\begin{tabular}{|c|c|c|c|c|c|}
\hline & $\begin{array}{l}\text { Carga } \\
\text { total del } \\
\text { jugador }\end{array}$ & $\begin{array}{l}\text { Carga del } \\
\text { jugader } \\
\text { por } \\
\text { minute }\end{array}$ & $\begin{array}{l}\text { Total de } \\
\text { esfluernos } \\
\text { explosivos' } \\
\text { min }\end{array}$ & $\begin{array}{l}\text { Esfuerzo } \\
\text { explosivos } \\
\text { altos }\end{array}$ & $\begin{array}{l}\text { Esfuerzos } \\
\text { explosiva } \\
\text { medios }\end{array}$ \\
\hline Jaege 1 & 36 & 4,33 & 12,75 & 12 & 21 \\
\hline Jaege 2 & 23 & 4,94 & 18,13 & 13 & 19 \\
\hline Jaego 3 & 29 & 5,21 & 17,73 & 15 & 22 \\
\hline Jaego 4 & 45 & 4,54 & 17,11 & 22 & 32 \\
\hline \multirow[t]{2}{*}{ Total } & 133 & 19,02 & 65,73 & 62 & 94 \\
\hline & $\begin{array}{l}\text { Esfuerzos } \\
\text { explosives } \\
\text { bajos }\end{array}$ & $\begin{array}{l}\text { Total de } \\
\text { saltos }\end{array}$ & $\begin{array}{l}\text { Saltos } \\
\text { medios } \\
(20- \\
40 \mathrm{~cm}) \\
\end{array}$ & $\begin{array}{l}\text { Salios } \\
\text { altos } \\
(>-40 \mathrm{~cm})\end{array}$ & \\
\hline Juego 1 & 72 & 1 & 0 & 0 & \\
\hline Juego 2 & 52 & 2 & 1 & 0 & \\
\hline Juego 3 & 62 & 2 & 0 & 0 & \\
\hline Juego 4 & 116 & 5 & 2 & 0 & \\
\hline Total & 302 & 10 & 3 & 0 & \\
\hline
\end{tabular}

Tabla 1. Resumen del anólisis tiempo-movimienta.

el jugador realizó un alto número (302) de esfuerzos explosivos bajos (movimientos locomotrices) comparando con los medios (peloteando con golpes de fondo) y esfuerzos explosivos altos (drives). Es interesante que hay muy pocos saltos, especialmente bajos durante la práctica de juego de partidos.

\section{COMENTARIO}

El período de preparación de las jugadoras proporcionó una oportunidad única de evaluar los perfiles de actividad que ocurren durante las actividades de alta intensidad prolongadas. El objetivo de este estudio consistió en investigar (1) los perfiles de actividad de un tenista de alto nivel, en períodos de práctica de aproximadamente 30 minutos, (2) la intensidad de la actividad durante períodos pre-definidos de práctica de juego de partidos. Los resultados de algunos estudios anteriores demuestran que comparado con jugadores más exitosos, los menos exitosos cubren una mayor distancia total, realizan más corridas de alta intensidad, y participan en mayores picos de esfuerzos repetidos de alta intensidad (Austin, Gabbett, Jenkins, 2011; Gabbett, 2012). Por lo tanto, algunos resultados sugieren que una mayor cantidad de actividades de alta intensidad y distancia total no se relaciona con el éxito en el deporte de elite (Gabbett, Jenkins, Abernethy, 2012; Hulin y cols. 2015). Hulin y cols. (2015) sugieren que el mayor número de colisiones tienen que ver con una mayor tasa de éxito en los equipo de la liga de rugby elite. Como resultado, nuestros hallazgos son importantes para los entrenadores y científicos deportivos cuando interpretan el análisis de tiempo- movimiento, nuestros resultados pueden indicar que la efectividad técnica y táctica del jugador pueden ser más determinantes de éxito que las mayores cargas de trabajo.

El reconocimiento de los factores fisiológicos y psicológicos que contribuyen para el desarrollo del rendimiento sería, por lo tanto, de particular valor para quienes administran las rutinas de entrenamiento (Terry, 2000). Este tipo de rastreo de las actividades de práctica, utilizando la tecnología GPS podría ser muy beneficioso para la aptitud física y para que los entrenadores de tenis eviten los síntomas de rendimiento deficiente o de sobre-entrenamiento. También, el uso de la tecnología puede ser útil para el análisis del rendimiento deportivo, y para que los entrenadores mejoren la calidad de la retroalimentación que entregan a sus atletas, y para incrementar la precisión en las mediciones de tiempo del rendimiento deportivo. Vale la pena también destacar, sin embargo, que el tamaño de la muestra tomada para este estudio es demasiado pequeño como para presentar una imagen clara, pero, se la considera importante y beneficiosa para medir estos cambios durante el período de preparación.

\section{CONCLUSIÓN}

El tenis actual requiere que el tenista cuente con un alto nivel de preparación. Al mismo tiempo, debemos tener conciencia de que el estrés por sobre entrenamiento puede generar "anquilosamiento" (Ryan, 1983; Morgan y cols., 1987). La tecnología GPS podría ser una herramienta beneficiosa para averiguar los perfiles de actividad de los tenistas, especialmente durante las sesiones de alta intensidad. Se necesitan estudios adicionales para identificar las 
intervenciones que puedan incrementar el rendimiento con el objetivo fundamental de lograr atletas más saludables.

\section{REFERENCIAS}

Austin, D.J., Gabbett, T.J., Jenkins, D.G. (2011). Repeated highintensity exercise in a professional rugby league. Journal of Strength and Conditioning Research, 25, 1898-1904. https://doi.org/10.1519/JSC.0b013e3181e83a5b

Duffield, R., Murphy, A., Kellett, A. Reid, M. (2014). Recovery From Repeated On-Court Tennis Sessions: Combining ColdWaterlmmersion, Compression, and Sleep Interventions. International Journal of Sports Physiology and Performance, 9:273-282. https://doi.org/10.1123/ijspp.2012-0359

Gabbett, T.J. (2012. Sprinting patterns of national rugby league competition. Journal of Strength and Conditioning Research, 26, 121-130. https://doi.org/10.1519/JSC.0b013e31821e4c60

Gabbett, T.J., Jenkins, D.G., Abernethy, B. (2012). Physical demands of professional rugby league training and competition using microtechnology. Journal of Science in Medicine and Sport, 15, 80-86. https://doi.org/10.1016/j.jsams.2011.07.004

Hagger, M., Chatzisarantis, N. (2005). Social Psychology of Exercise and Sport. McGraw-Hill International.

Hulin, B.T., Gabbett, T.J., Kearney, S., Corvo, A. (2015). Physical Demands of Match-Play in Successful and Less-Successful Elite Rugby League Teams. International Journal of Sports Physiology and Performance, 10, 703-710. https://doi.org/10.1123/ijspp.2014$\underline{0080}$

Morgan, W.P., Brown, D.R., Raglin, J.S, O'Connor, P.J., Ellickson, K.A. (1987). Psychological monitoring of overtraining and staleness. British Journal of Sports Medicine, 21,107-114. https://doi.org/10.1136/bjsm.21.3.107
Ryan, A.J. (1983). Overtraining in athletes: a roundtable. The physician and Sportsmedicine, 11, 93-100. https://doi.org/10.1080/00913847.1983.11708610

Terry, P. C. (2000). An overview of the relationship between mood and performance in sport. Australian Journal of Psychology, 52, S115.

CONTENIDO ITF ACADEMY RECOMENDADO (HAZ CLICK ABAJO)

\section{ITF Academy}

Derechos de Autor (c) 2017 Dario Novak y Magnus Norman

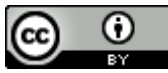

Este texto está protegido por una licencia CreativeCommons 4.0.

Usted es libre para Compartir -copiar y redistribuir el material en cualquier medio o formato-y Adaptar el documento - remezclar, transformar y crear a partir del material- para cualquier propósito, incluso para fines comerciales, siempre que cumpla la condición de:

Atribución: Usted debe dar crédito a la obra original de manera adecuada, proporcionar un enlace a la licencia, e indicar si se han realizado cambios. Puede hacerlo en cualquier forma razonable, pero no de forma tal que sugiera que tiene el apoyo del licenciante o lo recibe por el uso que hace de la obra.

$\underline{\text { Resumendelicencia - Textocompletodelalicencia }}$ 